VoL. 68 (2003) [155-159]

\title{
DENSE ORBITS AND COMPACTNESS OF GROUPS
}

\author{
W.H. Previts and T.S. Wu
}

\begin{abstract}
Using results from topological groups and topological dynamics for locally compact spaces, Aoki has shown that when a group automorphism of a locally compact totally disconnected group is ergodic under the Haar measure, the group is compact. We provide some remarks on Aoki's proof. Also, we present a new proof of his result using the structure of locally compact totally disconnected groups established by Willis.
\end{abstract}

\section{INTRODUCTION}

In [2], Halmos asked whether the Haar measure of a non-compact locally compact group can be ergodic under a group automorphism [2, p. 29]. This question was first answered for subclasses of Abelian groups and connected groups as follows.

THEOREM A. (Rajagopalan [5].) Let $X$ be a locally compact group with a left invariant Haar measure $\mu$ and let $f$ be a continuous automorphism of $X$. If $(X, f)$ is ergodic under $\mu$ and $X$ is Abelian, then $X$ is compact.

Theorem B. (Kaufman and Rajagopalan [3], Wu [8].) Let $X$ be a locally compact group with a left invariant Haar measure $\mu$ and let $f$ be a continuous automorphism of $X$. If $(X, f)$ is ergodic under $\mu$ and $X$ is connected, then $X$ is compact.

The two separate proofs of Theorem $B$ in [3] and [8] made use of the structure of locally compact connected groups and Lie groups. In both cases, the theorem was first proved for connected Lie groups. Then the result was extended to locally compact connected groups by using the fact that every locally compact connected group $G$ contains a maximal compact normal subgroup $N$ such that $G / N$ is a Lie group.

Aoki answered Halmos's question in [1] for the subclass of totally disconnected groups. In addition, Halmos's question has been completely answered with Aoki's result. More precisely,

THEOREM C. (Aoki [1].) Let $X$ be a locally compact group with a left invariant Haar measure $\mu$ and let $f$ be a continuous automorphism of $X$. Let $X_{0}$ denote the

Received 16th January, 2003

The authors would like to thank the referee for valuable suggestions and comments.

Copyright Clearance Centre, Inc. Serial-fee code: 0004-9727/03 \$A2.00+0.00. 
connected component of the identity in $X$. If $(X, f)$ is ergodic under $\mu$ and $X / X_{0}$ is compact, then $X$ is compact.

THEOREM 1. (Aoki [1].) Let $X$ be a locally compact group with a left invariant Haar measure $\mu$ and let $f$ be a continuous automorphism of $X$. If $(X, f)$ is ergodic under $\mu$ and $X$ is totally disconnected, then $X$ is compact.

THEOREM 2. Let $X$ be a locally compact group with a left invariant Haar measure $\mu$ and let $f$ be a continuous automorphism of $X$. If $(X, f)$ is ergodic under $\mu$, then $X$ is compact.

In contrast to proofs of Theorem B, Aoki's proof of Theorem 1 made use of results in topological dynamics. Theorem 1 was first reduced to the case when $X$ is metrisable and separable, $f$ is bicontinuous, and $(X, f)$ has a dense orbit. Also, it is only necessary to consider the case when $X$ is not discrete. Otherwise, if $X$ is discrete, then $X$ is trivial since $(X, f)$ has a dense orbit. In order to prove Theorem 1, Aoki established the following two propositions.

Propos ITION 3. (Aoki [1].) Let $X$ be a locally compact totally disconnected metric group with a left invariant metric function $d_{0}$ and let $f$ be a bicontinuous automorphism of $X$. If $(X, f)$ is topologically mixing and has the pseudo-orbit tracing property under $d_{0}$, then $X$ is compact.

Propos I TION 4. (Aoki [1].) Under the notations of Proposition 3, if $(X, f)$ has a dense orbit, then $(X, f)$ is topologically mixing and has the pseudo-orbit tracing property under $d_{0}$.

In this paper, we explain some statements that appear in the proofs of Propositions 3 and 4. We hope these notes will be useful for readers of Aoki's paper. Also, we present a new proof of Theorem 1 which uses the structure of locally compact totally disconnected groups established by Willis (see $[6,7])$.

\section{SOME NOTES ON AOKI'S PROOF}

The aim of this section is to clarify some statements that are found in the proofs of Propositions 3 and 4 . We begin with a lemma that will be used throughout the paper.

Lemma 1. Let $X$ be a non-discrete metric space, $f$ a homeomorphism of $X$, and suppose that $(X, f)$ has a dense orbit. If $A$ is a non-empty open and closed subset of $X$, and if either $f(A) \subseteq A$ or $A \subseteq f(A)$, then $A=f(A)=X$.

Proof: We consider the case where $f(A) \subseteq A$. Let $x$ be a point in $A$ whose orbit is dense. Since $X$ is not discrete, every point $y$ of $X$ is a limit of a sequence of $f^{n_{i}}(x)$, where we may assume that all of the $n_{i}>0$ or all of the $n_{i}<0$, that is, $y \in \omega(x)$ or $y \in \alpha(x)$. Note that both $\omega(x)$ and $\alpha(x)$ are closed invariant subsets of $X$. 
Suppose $x \in \omega(x)$. Since $x \in A$ and $f(A) \subseteq A, f^{n}(x) \in A$ for all $n>0$. Let $l>0$ and consider $f^{-l}(x)$. Since $x=\lim _{n_{i} \rightarrow \infty} f^{n_{i}}(x), n_{i}>0$, we have $f^{-l}(x)$ $=f^{-l}\left(\lim _{n_{i} \rightarrow \infty} f^{n_{i}}(x)\right)=\lim _{n_{i} \rightarrow \infty} f^{n_{i}-l}(x) \in A$, since $f^{n_{i}-l}(x) \in A$ for all $n_{i}>l$ and $A$ is closed. Thus $f^{n}(x) \in A$ for all $n \in \mathbb{Z}$. Therefore $\overline{O(x)}=\left\{\overline{f^{n}(x): n \in \mathbb{Z}}\right\} \subseteq f(A) \subseteq A$. Since $\overline{O(x)}=X$, we can conclude that $X=f(A)=A$.

Now suppose $x \in \alpha(x)$. Since $f(A) \subseteq A, A \subseteq f^{-1}(A)$, so $f^{-1}(X \backslash A) \subseteq X \backslash A$. Let $B=X \backslash A$. Then $B$ is both open and closed, and $f^{-1}(B) \subseteq B$. We may assume that $B \neq \emptyset$. Otherwise, $A=X$. Then $f^{l}(x) \in B$ for some $l \in \mathbb{Z}$. Write $y=f^{l}(x)$. Since $x=\lim _{n_{i} \rightarrow-\infty} f^{n_{i}}(x), n_{i}<0$, we have

$$
y=f^{l}(x)=f^{l}\left(\lim _{n_{i} \rightarrow-\infty} f^{n_{i}}(x)\right)=\lim _{n_{i} \rightarrow-\infty} f^{n_{i}+l}(x)=\lim _{n_{i} \rightarrow-\infty} f^{n_{i}}\left(f^{l}(x)\right) .
$$

Thus $y \in \alpha(y)$. Write $h=f^{-1}$. Then $h(B) \subseteq B, y \in \omega(y)$ (with respect to $h$ ), and $y$ has a dense orbit (with respect to $h$ ). Thus $B=h(B)=X$. This implies that $A=\emptyset$, which is a contradiction.

Now we explain four statements that appear in the proofs of Propositions 3 and 4. We use the same notation as Aoki.

(1) In [1, Proposition 3, Claim 1.6] Aoki states: "Since $(X, g)$ has a dense orbit, it follows that $K \backslash g(K)=\emptyset$, which implies $K=X$ ". This follows by Lemma 1, since $K$ is both open and closed and $g(K) \subseteq K$.

(2) In [1, p. 5 , the second paragraph of the proof of Proposition 4], Aoki writes: "If the left coset space $X / A$ is discrete, then $A$ is open in $X$. Hence either $X$ is discrete or $f(A)=A$ holds". We notice that $f(A) \subseteq A$. To see that $f(A)=A$, use Lemma 1 , since $A$ is an open and closed subgroup.

(3) In [1, proof of Proposition 4, Claim 2.10], Aoki states: "Let $\delta$ ' be the number such that if $d_{1}^{\prime}\left(f^{-k}(x) A, f^{-k}(y) A\right)<\delta^{\prime}$, then $d_{4}\left(\left\{f^{-i}(x) A\right\}_{1}^{\infty}\left\{f^{-i}(y) A\right\}_{1}^{\infty}\right)<\varepsilon$. We now explain how to choose $\delta^{\prime}$.

Choose a positive integer $k$ such that $\sum_{i=k+1}^{\infty} 1 / 2^{i}<\varepsilon / 2$.

We note that if $d_{1}^{\prime}\left(f^{-i}(x) A, f^{-i}(y) A\right)<\alpha$ for some $\alpha>0$, then $d_{1}^{\prime}\left(f^{-i}\left(x^{-1} y\right) A\right.$, $A)<\alpha$, that is, $f^{-i}\left(x^{-1} y\right) A \in B_{\alpha}(A)$, where $B_{\alpha}(A)$ denotes the ball of radius $\alpha$ and centre $A$.

Since $g: X / A \rightarrow X / A$, defined by $g(x A)=f(x) A$, is continuous, given any $\beta>0$ there exists $\gamma>0$ such that $g\left(B_{\gamma}(A)\right) \subseteq B_{\beta}(A)$. Thus if $f^{-i}\left(x^{-1} y\right) A \in B_{\gamma}(A)$, that is, $d_{1}^{\prime}\left(f^{-i}(x) A, f^{-i}(y) A\right)<\gamma$, then $d_{1}^{\prime}\left(f^{-i+1}(x) A, f^{-i+1}(y) A\right)<\beta$.

To construct $\delta^{\prime}$, let $\beta_{1}=\varepsilon / 2^{k}$. Then there exists $\beta_{2}>0$ such that $g\left(B_{\beta_{2}}(A)\right)$ $\subseteq B_{\beta_{1}}(A)$. Since $\beta_{2}>0$, there exists $\beta_{3}>0$ such that $g\left(B_{\beta_{3}}(A)\right) \subseteq B_{\beta_{2}}(A)$. 
Now we repeat this argument $(k-1)$ times. Note that each time we choose the $\beta_{i}$, we have to make sure that the $\beta_{i}$ is less than $\varepsilon / 2^{k}$ because we have to add up $\sum_{i=1}^{k}\left(1 / 2^{i}\right) d_{1}^{\prime}\left(f^{-i}(x) A, f^{-i}(y) A\right)$. Finally, let $\delta^{\prime}=\beta_{k}$.

(4) In [1, p. 10] Aoki states: "By Claims 2.7 and 2.11, the set of all periodic points of $h$, per $(h)$, is dense in $X / B$ ". Here we provide a proof of this statement which was not included in his paper. The proof is due to Aoki.

LEMMA 2. Let $(X, d)$ be a metric space, $f$ a homeomorphism of $X$, and suppose that $(X, f)$ has a dense orbit. If $(X, f)$ is expansive and has the pseudo-orbit tracing property, then the set of periodic points, $\operatorname{per}(f)$, is dense in $X$.

Proof: Let $x_{0}$ be a point such that $O\left(x_{0}\right)$ is dense in $X$. Let $\varepsilon>0$ be an expansive constant for $f$. Since $f$ has the pseudo-orbit tracing property, there exists a $\delta>0$ such that each $\delta$-pseudo-orbit can be $\varepsilon / 2$ traced to some point in $X$. For any $x \in X$, we can find points $f^{n+1}\left(x_{0}\right), f^{m}\left(x_{0}\right)$ such that $d\left(f^{n+1}\left(x_{0}\right), f(x)\right)$ $<\delta$ and $d\left(f^{m}\left(x_{0}\right), x\right)<\delta$. We can assume that $n+1<m$. Then we construct a periodic $\delta$-pseudo-orbit $\left\{x, f^{n+1}\left(x_{0}\right), \ldots, f^{m-1}\left(x_{0}\right), x\right\}=\left\{z_{0}, z_{1}, \ldots, z_{m-n-1}, z_{0}\right\}$. By the pseudo-orbit tracing property, there is a tracing point $z \in X$ such that $d\left(f^{i}(z), z_{i}\right)$ $<\varepsilon / 2(i \in \mathbb{Z})$. Thus $d\left(f^{m-n+i}(z), f^{i}(z)\right)<\varepsilon(i \in \mathbb{Z})$, and therefore $f^{m-n}(z)=z$ because of the expansiveness of $f$. Therefore $\operatorname{per}(f)$ is dense in $X$.

\section{A NEW PROOF OF THEOREM 1}

In recent years, Willis has developed a structure of locally compact totally disconnected groups. An important feature of this theory is the notion of a tidy subgroup (see $[4,6,7])$. In this section we provide a new proof of Theorem 1 using tidy subgroups. Here we recall a few results of Willis regarding these subgroups.

TheOREM 3. (Willis $[6,7]$.) Let $G$ be a totally disconnected locally compact group and let $f$ be a bicontinuous automorphism of $G$. Then there is a compact open subgroup $U$ of $G$ such that, putting $U_{+}=\bigcap_{n=0}^{\infty} f^{n}(U)$ and $U_{-}=\bigcap_{n=0}^{\infty} f^{-n}(U)$, we have:

(i) $U=U_{+} U_{-}=U_{-} U_{+}$, and

(ii) $\bigcup_{n=0}^{\infty} f^{n}\left(U_{+}\right)$and $\bigcup_{n=0}^{\infty} f^{-n}\left(U_{-}\right)$are closed in $G$.

A compact open subgroup $U$ satisfying properties (i) and (ii) of Theorem 3 is said to be tidy for $f$.

Theorem 4. (Willis [6, Proposition 1].) Let $G$ be a totally disconnected locally compact group and let $U$ be a compact open subgroup of $G$ which is tidy for $f$. Then $\bigcup_{n=0}^{\infty} f^{n}(U)$ and $\bigcup_{n=0}^{\infty} f^{-n}(U)$ are open and closed subsets of $G$. 
Here we provide a new proof of Theorem 1 which parallels the last paragraph of Aoki's Proposition 3 (see [1]).

Proof of Theorem 1: As in Aoki's paper [1], we can reduce Theorem 1 to the case when $X$ is metrisable and separable, $f$ is bicontinuous, $(X, f)$ has a dense orbit, and $X$ is not discrete. Let $U$ be a compact open subgroup of $X$ which is tidy for $f$. Define $U_{m}=U \cup f(U) \cup \ldots \cup f^{m}(U)$. By Theorem 4, $U^{*}=\bigcup_{m=0}^{\infty} U_{m}$ is an open and closed subset of $X$. Clearly $f\left(U^{*}\right) \subseteq U^{*}$. Thus by Lemma $1, f\left(U^{*}\right)=U^{*}=X$.

Because $f^{-1}(U)$ is compact, there exists $m \geqslant 0$ such that $f^{-1}(U) \subseteq U_{m}$. Hence $U_{m} \subseteq U_{m+1}=U \cup f\left(U_{m}\right)=f\left(U_{m}\right)$. Since $U_{m}$ is an open and closed subset of $X$, by Lemma 1, we have that $U_{m}=f\left(U_{m}\right)=X$. Thus $X$ is compact.

\section{REFERENCES}

[1] N. Aoki, 'Dense orbits of automorphisms and compactness of groups', Topology Appl. 20 (1985), 1-15.

[2] P.R. Halmos, Lectures on ergodic theory (Publ. Math. Soc. Japan, Tokyo, 1956).

[3] R. Kaufman and M. Rajagopalan, 'On automorphisms of a locally compact group', Mich. Math. J. 13 (1966), 373-374.

[4] W.H. Previts and T.S. Wu, 'On tidy subgroups of locally compact totally disconnected groups', Bull. Austral. Math. Soc. 65 (2002), 485-490.

[5] M. Rajagopalan, 'Ergodic properties of automorphisms of a locally compact group', Proc. Amer. Math. Soc. 17 (1966), 372-376.

[6] G. Willis, 'The structure of totally disconnected, locally compact groups', Math. Ann. 300 (1994), 341-363.

[7] G. Willis, 'Further properties of the scale function on a totally disconnected group', $J$. Algebra 237 (2001), 142-164.

[8] T.S. Wu, 'Continuous automorphisms on locally compact groups', Math. Z. 96 (1967), 256-258.

Department of Mathematics

Lakeland Community College

7700 Clocktower Drive

Kirtland OH 44094

United States of America
Department of Mathematics

Case Western Reserve University

Cleveland OH 44106

United States of America 